\section{Determinantes sociais e biológicos da cárie dentária em crianças de 6 anos de idade: um estudo transversal aninhado numa coorte de nascidos vivos no Sul do Brasil *}

\section{Effects of Social and biological factors on dental caries in 6-year-old children: a cross sectional study nested in a birth cohort in Southern Brazil}

\author{
Marco Aurélio Peres \\ Departamento de Epidemiologia \\ Faculdade de Saúde Pública \\ Universidade de São Paulo \\ Departamento de Saúde Pública do Centro de Ciências da Saúde \\ Universidade Federal de Santa Catarina \\ Campus Universitário, Trindade \\ 88010-970 - Florianópolis, SC, Brasil \\ peresp@repensul.ufsc.br \\ Maria do Rosário D. O. Latorre \\ Departamento de Epidemiologia \\ Faculdade de Saúde Pública \\ Universidade de São Paulo \\ Aubrey Sheiham \\ Department of Epidemiology and Public Health \\ Royal Free and University College London \\ London, UK \\ Karen Glazer Peres \\ Departamento de Epidemiologia \\ Faculdade de Saúde Pública \\ Universidade de São Paulo \\ Universidade do Sul de Santa Catarina \\ Fernando Celso Barros \\ Centro Latinoamericano de Perinatologia \\ Montevideo - Uruguai \\ Pedro Gonzales Hernandez \\ Faculdade de Odontologia \\ Universidade Federal de Pelotas, RS \\ Angela Maria Nunes Maas \\ Faculdade de Odontologia \\ Universidade Federal de Pelotas, RS \\ Ana Regina Romano \\ Faculdade de Odontologia \\ Universidade Federal de Pelotas, RS

\section{Cesar Gomes Victora} \\ Centro de Pesquisas Epidemiológicas \\ Departamento de Medicina Social \\ Universidade Federal de Pelotas
}

\section{Resumo}

Objetivo: O objetivo deste estudo foi investigar os determinantes sociais e biológicos, medidos no período perinatal e na infância, sobre a ocorrência de cárie dentária em crianças de 6 anos de idade. Métodos: Foi realizado um estudo transversal de cárie dentária em 1999, em uma subamostra de 400 crianças pertencentes a uma coorte de nascidos vivos iniciada em 1993, em Pelotas, RS. Os índices e critérios de diagnóstico adotados foram os preconizados pela Organização Mundial da Saúde (1997). Os resultados do estudo de cárie foram inseridos no banco de dados da coorte, que continha informações sobre as condições sociais e de saúde coletadas ao nascimento, no primeiro, terceiro, sexto e décimo segundo meses e no sexto ano de vida da criança. O índice de cárie (ceo-d $=0 \mathrm{ou} \geq 1$ ) foi a variável dependente. Foram realizadas análises univariada e de regressão logística múltipla não condicional, tendo como base um modelo teórico hierárquico de determinação. Resultados: Escolaridade materna abaixo ou igual a 8 anos, renda familiar menor que 6 salários mínimos, não freqüentar pré-escola e consumo de doces pelo menos uma vez ao dia aos 6 anos de idade foram fatores de risco à cárie. Conclusões: Os fatores de risco sociais, como baixa escolaridade materna e baixa renda familiar, não freqüentar a pré-escola e dieta inadequada são comuns à cárie dentária e outras doenças e agravos infantis, sugerindo que medidas de intervenção dirigidas a estes fatores seriam mais adequadas à prevenção da cárie do que medidas específicas.

Palavras-chave: Cárie dentária. Estudo de coorte. Período perinatal e primeira infância. Classe social. Fatores de risco.

"Baseado na tese de doutorado de Marco Aurélio Peres. Curso de pós-graduação em Saúde Pública - área de concentração Epidemiologia - da Faculdade de Saúde Pública da USP, 2002. 
Abstract

Objective: The aim of this study was to investigate the relationship between the social and biological conditions experienced in early life and dental caries in 6-year-old children, using a life course approach. Methods: An oral health cross-sectional study was nested in a birth cohort study initiated in Pelotas, Brazil in 1993. In 1999, a sample of 400 6-year-old children was selected from the cohort. The criteria of the World Health Organization (1997) were used for dental caries. The results from the dental caries study were analyzed in relation to the socioeconomic and morbidity data collected at birth, and at 1, 3, 6 and 12 months, and the sixth year of life. Dental caries were measured through the decayed, missing and filled index (dmft). Unconditional univariate and multiple logistic regression analyses were performed according to a conceptual model. Results: Risk factors for the occurrence of dental caries included maternal education equal or less than 8 years, family income lower than six minimum wages, not attending day care, and consumption of sweets at least once a day at age 6. Conclusions: Strategies for oral health care should include inter-sector approaches for health promotion based on a population strategy and a common risk approach.

Keywords: Dental caries. Cohort. Early life. Social class. Risk factors.

\section{Introdução}

A cárie dentária é a doença mais comum em crianças da região das Américas, constituindo um aspecto crítico das condições gerais de saúde devido ao seu peso na carga de doenças, e ao seu impacto na qualidade de vida por ser causa de dor e sofrimento ${ }^{1}$. Além disso, os altos custos de tratamento e a possibilidade de utilização de medidas preventivas efetivas contribuem para ela ser considerada um importante problema de saúde pública ${ }^{1}$.

A cárie dentária é uma doença crônica resultante da dissolução mineral dos tecidos dentários proveniente da produção de ácidos produzidos por bactérias quando estas metabolizam carboidratos, em especial a sacarose, oriundos da dieta. Apesar da etiologia da cárie ser bem conhecida, muitos aspectos relativos ao papel dos fatores sociais e biológicos nos primeiros anos de vida continuam obscuros ${ }^{2}$. Alguns pesquisadores têm identificado a influência de fenômenos que ocorrem no período perinatal e da primeira infância na determinação de cárie anos mais tarde, como baixo peso ao nascer, doenças e sintomas apresentados nos primeiros anos e déficit na relação entre altura e idade no primeiro ano de vida, indicador síntese do estado nutricional infantil ${ }^{3,4}$.

No início da década de 90, foram propostos modelos etiológicos baseados na hipótese de associação entre condições e doenças infantis com a ocorrência de doenças futuras, inclusive em adultos 5 . A tese central desta proposição é a de que a nutrição e o ambiente durante o período fetal e infantil, assim como a exposição a infecções durante a infância, gerariam o aparecimento de doenças muitos anos mais tarde, sendo este processo denominado por Barker de "programação biológica" ${ }^{5}$. Dentre as possíveis relações existentes destacam-se aquelas entre os problemas nutricionais e ambientais ocorridos durante a vida intra-uterina e a infância, e as doenças coronarianas, diabetes, hipertensão e aumento das taxas de colesterol na vida adulta.

Sem deixar de lado a importância da influência dos primórdios da vida em relação 
à ocorrência de doenças futuras, foi formulada outra teoria, esta baseada no acúmulo de riscos através da vida e na importância do período infantil. Parte-se da hipótese de que as doenças crônicas são decorrentes de um acúmulo de riscos no curso da vida, como os riscos sociais, as doenças, o ambiente e os comportamentos ${ }^{6}$. Esta teoria (life course approach) não requer a noção chave, expressa na teoria de Barker, de período crítico, eliminando assim o seu caráter determinista. Esta teoria sugere que, através do curso da vida, exposições prejudiciais à saúde são acumuladas através de fatores relativos ao desenvolvimento infantil, como baixo peso ao nascer, episódios de doenças e condições sociais, ambientais e comportamentais adversas. Se estes insultos são numerosos ao longo do curso da vida, o risco de adquirir doenças crônicas aumenta. Kuh e Ben-Shlomo ${ }^{6}$ identificaram três tipos de processos nos quais as condições ocorridas em período muito precoce da vida poderiam afetar a saúde e o comportamento anos mais tarde. Estes processos freqüentemente interagem uns com os outros. São eles:

- efeito latente da exposição ambiental no início da vida na saúde anos mais tarde, por exemplo, quando apresenta-se uma desvantagem biológica específica ocorrida em um período muito sensível no início da infância, como nascer com baixo peso ou apresentar retardo no desenvolvimento;

- efeitos cumulativos ao longo do tempo, dependendo da sua intensidade e duração, podem afetar a saúde anos mais tarde; e

- uma trajetória de complexa interação entre indivíduo e ambiente influenciando o curso da vida ${ }^{6}$.

Como muitos fatores de risco são comuns, tanto para doenças sistêmicas crônicas quanto para doenças bucais ${ }^{7}$, é razoável supor que teorias explicativas propostas para as doenças crônicas possam ser aplicadas à saúde bucal. Desta forma, seria possível elucidar quais e quando os fatores de risco ocorrem, indicando o tempo mais apropri- ado para a adoção de medidas preventivas e assistenciais, além de contribuir para a adoção de abordagens multiprofissionais e intersetoriais.

O objetivo deste trabalho foi identificar os fatores de risco sociais e biológicos relativos ao período perinatal e da primeira infância para a ocorrência de cárie em crianças de 6 anos de idade.

\section{Métodos}

Este estudo insere-se em uma coorte de nascidos vivos iniciada em 1993 na cidade de Pelotas, Rio Grande do Sul, cujo objetivo foi estudar a saúde perinatal e infantil das crianças. ${ }^{8}$.

Em 1999, quando as crianças da coorte completaram 6 anos de idade, foi desenvolvido o Estudo de Saúde Bucal (ESB), um subprojeto do estudo de coorte. O ESB foi um estudo transversal no qual foram investigadas diferentes condições e doenças bucais.

A partir do universo das 5.249 crianças nascidas vivas em Pelotas, RS, em 1993, foi obtida uma amostra de $20 \%$ para o acompanhamento infantil, incluindo todos os nascidos de baixo peso. Em 1998, 1.273 crianças da amostra foram localizadas. Desta amostra foi obtida uma subamostra de 400 crianças para o ESB. Para o cálculo considerouse a prevalência de cárie de $80 \%$ para as crianças expostas aos fatores de risco e $65 \%$ para as não expostas, adotando-se um nível de significância de $5 \%$ e um poder do teste igual a $80 \%$, totalizando 302 crianças. Como outros desfechos relativos às oclusopatias foram investigados, adotou-se o maior tamanho da amostra, o que totalizou 400 crianças, já se adicionando $10 \%$ a mais de crianças a fim de compensar não respostas e perdas. Para a seleção da amostra do ESB foi adotado o método casual simples.

Como foram incluídos todos os nascidos de baixo peso, perfazendo $28,7 \%$ da amostra (103 crianças), todas as análises decorrentes foram ponderadas de forma a representar a verdadeira proporção de nascidos de baixo peso. Detalhes sobre os méto- 
dos empregados no estudo de coorte encontram-se em outra publicação ${ }^{8}$.

As coletas de dados sociais, médicos e antropométricos foram realizadas ao nascimento da criança, no primeiro, terceiro, sexto e décimo segundo meses, e no sexto ano de vida da criança.

O ESB foi composto de um questionário que apresentava perguntas relativas aos hábitos, comportamentos e acesso aos serviços, e de exames odontológicos.

Antes dos exames houve a calibração dos examinadores com três entrevistadores e três examinadores experientes em estudos epidemiológicos de saúde bucal, sendo um dos autores deste estudo (MAP) o orientador do treinamento. Através da estatística kappa, mediu-se a reprodutibilidade diagnóstica dos examinadores, realizada dente a dente, sendo o menor valor obtido igual a 0,75 , e a maioria dos valores iguais a 1,0 .

As crianças da amostra foram visitadas no domicílio pela equipe da pesquisa do ESB entre os meses de dezembro de 1998 a junho de 1999. Nesta ocasião foi realizada a entrevista com os pais ou responsáveis e o exame odontológico, tendo sido informados os objetivos do presente estudo. $\mathrm{O}$ projeto foi aprovado pelo Comitê de Ética em Pesquisa da Universidade Federal de Pelotas. Quando houve impossibilidade da realização da entrevista e do exame odontológico foi agendada uma nova visita dos entrevistadores/examinadores. Até quatro visitas foram realizadas antes de se considerar a criança como uma perda, incluindo pelo menos uma vista noturna e uma nos fins de semana. As crianças com necessidades de tratamento odontológico foram encaminhadas para a Faculdade de Odontologia da UFPel.

Foram realizados exames odontológicos com o objetivo de se conhecer $o$ ataque de cárie dentária à dentição decídua, através do índice ceo-d (dentes cariados, perdidos em virtude da cárie e restaurados) modificado pela Organização Mundial da Saúde ${ }^{9}$.

Os exames foram realizados com o auxílio de uma lanterna portátil, em espaço aberto, com o examinador e o examinado senta- dos. As normas de biossegurança foram rigorosamente respeitadas pela equipe de campo.

A variável dependente foi o índice ceo-d, dicotomizado em zero ou maior ou igual a 1. As variáveis independentes foram analisadas em quatro blocos (Quadro 1).

O bloco 1 foi constituído pelas variáveis socioeconômicas, tais como a classe social na forma como foi operacionalizada por Lombardi et $\mathrm{al}^{10}$; a renda familiar aferida pela soma dos salários, pelos rendimentos ou aposentadorias de todas as pessoas da residência da criança pesquisada convertidos em salários mínimos vigentes na época; a escolaridade, em anos, dos pais e das mães na época do nascimento da criança; e o acesso a água encanada no domicilio na ocasião do nascimento da criança e aos 12 meses de idade.

O segundo bloco foi formado pelas variáveis que representaram as características do trabalho dos pais, tais como o número de meses que a mãe trabalhou durante a gravidez, a situação de trabalho do pai quando do nascimento da criança, e a situação de trabalho da mãe aos 12 meses e no sexto ano de vida da criança.

As variáveis relativas às características da criança e ao seu crescimento e desenvolvimento compuseram o terceiro bloco e foram as seguintes: sexo da criança, o número de dentes presentes aos 12 meses, o peso ao nascer, a relação entre altura e idade aos 12 meses, a relação entre peso e idade aos 12 meses, a relação entre peso e altura aos 12 meses, estas medidas antropométricas seguindo a classificação do National Center for Health Statistics ${ }^{11}$. Além destas, o tempo de amamentação; o uso de mamadeira noturna; o uso crônico de medicamentos, definido como o de pelo menos durante 30 dias todos os dias; e episódios de hospitalização também foram investigados.

O quarto conjunto de variáveis foi composto pelas variáveis que expressavam os cuidados com a criança e os hábitos relativos à saúde bucal, tais como a freqüência da criança à pré-escola aos 6 e 12 meses e no sexto ano de vida, acesso a consulta odontológica, 
Quadro 1 - Modelo teórico da hierarquia entre as variáveis estudadas.

Chart 1 - Theoretical hierarchy model for variables studied

\begin{tabular}{ll}
\hline & Bloco socioeconômico \\
\hline Variáveis & Categorias \\
\hline Classe social & Burguesia + NPB; PBT; Proletariado \\
Escolaridade dos pais & 8 anos; $\leq 8$ anos \\
Renda Familiar & $>6 \mathrm{SM} ; 1,1$ a $6 \mathrm{SM} ; \leq 1 \mathrm{SM}$ \\
Presença de água encanada ao nascimento & Sim; Não \\
Presença de água encanada aos 12 meses & Sim, em casa; Sim, no pátio; Não \\
\hline & Bloco Trabalho dos pais \\
\hline Variáveis & Categorias \\
\hline Tempo de trabalho da mãe na gravidez & 8 a 9 meses; 1 a 7 meses; Não trabalhou \\
Situação de trabalho do pai na gravidez & Trabalhando; Desempregado/afastado \\
Trabalho da mãe aos 12 meses & Sim; Não \\
Trabalho da mãe no $6^{\circ}$ ano de vida & Todos os meses do último ano; Alguns meses; Não trabalhou \\
\hline
\end{tabular}

Bloco Crescimento e desenvolvimento infantil

\begin{tabular}{ll}
\hline Variáveis & Categorias \\
\hline Peso ao nascer & Adequado; Baixo \\
Altura/idade aos 12 meses & Adequada; Inadequada \\
Peso/altura aos 12 meses & Adequada; Inadequada \\
Peso/idade aos 12 meses & Adequada; Inadequada \\
Número de dentes aos 12 meses & $\leq 5 ;>5$ \\
Medicamentos crônicos $1^{\circ}$ ao $6^{\circ}$ ano de vida & Não; Sim \\
Hospitalizações na vida & Não; Sim \\
Duração total da amamentação & $\geq 9$ meses; 4 a 8,9 meses; 1 a 3,9 meses; $<1$ mês \\
Uso de mamadeira noturna & Não; Sim \\
\hline
\end{tabular}

\section{Bloco Cuidado com a criança, comportamentos e hábitos}

Variáveis Categorias

Freqüência à creche aos 6 meses

Sim; Não

Freqüência à creche aos 12 meses Sim; Não

Freqüência à pré-escola no $6^{\circ}$ ano de vida Sim; Não

Acesso ao dentista no $6^{\circ}$ ano de vida e aos 6 anos Sim; Não

Número de vezes que escova os dentes aos $\quad \geq 3 \mathrm{X}$ ao dia; 1 a $2 \mathrm{X}$ ao dia; $<1 \mathrm{X}$ ao dia

6 anos de idade

Época em que iniciou a escovação Qdo erupcionaram $1^{\text {os }}$ dentes; Após 1 ano

Ajuda de adulto na escovação Sim; Não

Uso de fio dental aos 6 anos Sim; Não

Consumo de doces aos 6 anos $\quad<1 \mathrm{X}$ ao dia; $\geq 1 \mathrm{X}$ ao dia todo dia

Costuma mascar chicletes aos 6 anos de idade Não; Sim

Bebida mais consumida aos 6 anos de idade Água; Outra

Ações educativas de S. Bucal na escola Sim; Não

NPB: Nova pequena burguesia; PBT: Pequena burguesia tradicional; SM: salário mínimo/[NPB: new middle class; PBT: traditional middle class; SM: minimum wage] 
freqüência de escovação dentária, uso do fio dental aos 6 anos de idade, época em que iniciou a escovação, ajuda de adulto na escovação, consumo de doces aos 6 anos, costume de mascar chicletes aos 6 anos de idade, a bebida mais consumida aos 6 anos de idade, e se participou de ações educativas de saúde bucal na escola aos 6 anos de idade.

Os dados foram digitados no programa Epiinfo versão 6.04 e posteriormente exportados para o programa SPSS para Windows, versão 10.0. Foram realizados testes de associação pelo qui-quadrado para avaliar o relacionamento entre a variável dependente e as variáveis independentes, estimando-se as razões de chance (Odds Ratio) e respectivos intervalos de confiança.

$\mathrm{Na}$ análise dos fatores de risco para a ocorrência de cárie, utilizou-se um modelo hierárquico de determinação (Quadro 1). No modelo proposto constituíram-se nos determinantes distais as variáveis que compuseram o bloco socioeconômico. Estas condicionaram os demais blocos de fatores de risco: relativos ao trabalho, ao crescimento e desenvolvimento infantil (determinantes intermediários) e aos cuidados com a criança, hábitos relativos à saúde bucal e consumo de doces (determinantes proximais).

As variáveis com nível de significância p < 0,20 observadas na análise univariada, entraram na análise de regressão logística múltipla $^{12}$ dentro de cada bloco de variáveis. Utilizou-se o procedimento stepwise forward selection, ou seja, iniciou-se o modelo pela variável com maior significância estatística na análise univariada, e a seguir foram acrescentadas as outras variáveis, uma a uma, por ordem decrescente de significância estatística. A nova variável foi mantida no modelo múltiplo caso fosse estatisticamente significativa $(p<0,05)$ e/ou fosse variável de controle.

As variáveis sexo e número de dentes presentes aos 12 meses de idade foram utilizadas como controles, independentemente da significância estatística apresentada.

Foram selecionadas para compor a análise de regressão logística hierarquizada (modelo final) as variáveis de cada bloco que permaneceram com significância estatística
( $\mathrm{p}<0,05)$ quando da análise de regressão múltipla interna a cada bloco.

No modelo hierárquico, as variáveis do bloco socioeconômico foram as primeiras a serem incluídas e permaneceram como fatores de ajuste para os blocos hierarquicamente inferiores, o mesmo acontecendo com as variáveis dos blocos seguintes. Para testar o ajuste do modelo final foi empregado o teste de Hosmer e Lemeshow ${ }^{12}$.

\section{Resultados}

A taxa de resposta do estudo foi de $89,8 \%$ $(\mathrm{n}=359)$ em relação às crianças visitadas em 1998, sendo as perdas decorrentes, principalmente, de mudança da família para outro município. Foram testadas associações entre algumas importantes variáveis estudadas, tais como a escolaridade dos pais, o sexo da criança e o peso ao nascer no grupo efetivamente estudado (amostra) e no grupo constituído pelas perdas. Não foram identificadas diferenças estatisticamente significantes entre os dois grupos.

A prevalência de cárie foi de $62,5 \%$ e o índice ceo-d médio foi igual a 3,38, sendo a maior parte do índice constituído pelo componente cariado (97\%). Apenas 0,02 dentes em média (0,59\% do índice) constituíram-se dentes extraídos devido à cárie, enquanto 0,08 dentes em média (2,37\% do índice) encontravam-se restaurados (dados não apresentados).

A Tabela 1 apresenta a análise univariada e múltipla do bloco composto pelas variáveis socioeconômicas. Observa-se que renda familiar, classe social, escolaridade do pai e da mãe foram fortemente associadas à ocorrência de cárie $(\mathrm{p}<0,001)$. Após o ajuste dentro do bloco socioeconômico, a escolaridade da mãe e a renda familiar mantiveram-se associadas à ocorrência de cárie $(\mathrm{p}=0,012 \mathrm{e}$ $\mathrm{p}=0,032$, respectivamente). Crianças cujas mães estudaram 8 anos ou menos e cuja renda familiar foi menor que 6 salários mínimos tiveram maior risco de apresentar cárie. Classe social, escolaridade do pai e presença de água encanada ao nascimento perderam a significância estatística após o ajuste. 
Tabela 1 - Distribuição da proporção ponderada (\% p) da população segundo o ataque de cárie dentária (ceo-d $\geq 1$ ) e condições socioeconômicas em crianças de 6 anos de idade. Pelotas, RS, Brasil, 1999.

Table 1 - Weighed proportion (\% p) of the population according to the dental caries index (ceo- $d \geq 1$ ) and socioeconomic conditions in 6-year-old children. Pelotas, RS, Brasil, 1999.

\begin{tabular}{|c|c|c|c|c|c|}
\hline Variáveis/categorias & ceo- $d \geq 1 \% p$ & $\mathrm{OR}^{\mathrm{b}}\left[\mathrm{IC}_{95 \%}(\mathrm{OR})\right]$ & Nível P & $\mathrm{OR}^{\mathrm{a}}\left[\mathrm{IC} \mathrm{C}_{95 \%}(\mathrm{OR})\right]$ & Nível P \\
\hline Classe social & & & $<0,001$ & & \# \\
\hline Burguesia + NPB & 18,2 & 1,0 & & $\#$ & \\
\hline PBT & 65,6 & $8,2[2,5-27,0]$ & & & \\
\hline Proletariado & 63,9 & $7,7[2,5-23,2]$ & & & \\
\hline Escolaridade do pai & & & 0,001 & & $\#$ \\
\hline$>8$ anos & 48,0 & 1,0 & & $\#$ & \\
\hline$\leq 8$ anos & 67,9 & $2,3[1,4-3,8]$ & & & \\
\hline Escolaridade da mãe & & & $<0,001$ & & 0,012 \\
\hline$>8$ anos & 45,4 & 1,0 & & 1,0 & \\
\hline$\leq 8$ anos & 68,7 & $2,6[1,6-4,2]$ & & $2,0[1,2-3,3]$ & \\
\hline Renda familiar & & & $<0,001$ & & 0,032 \\
\hline$>6 \mathrm{SM}$ & 38,9 & 1,0 & & 1,0 & \\
\hline 1,1 a 6 SM & 64,8 & $2,9[1,6-5,3]$ & & $2,1[1,1-4,1]$ & \\
\hline$\leq 1 \mathrm{SM}$ & 74,2 & $4,5[2,0-10,0]$ & & $3,0[1,3-7,6]$ & \\
\hline Água encanada no nascimento & & & 0,051 & & $\#$ \\
\hline Sim & 60,1 & 1,0 & & $\#$ & \\
\hline Não & 74,1 & $1,9[1,0-3,7]$ & & & \\
\hline Água encanada aos 12 meses & & & 0,497 & & * \\
\hline Sim, em casa & 61,8 & 1,0 & & * & \\
\hline Sim, no pátio & 75,0 & $1,7[0,6-4,8]$ & & & \\
\hline Não & 61,5 & $0,9[0,3-2,9]$ & & & \\
\hline
\end{tabular}

$\mathrm{OR}^{\text {b: }}$ Valores brutos; OR ${ }^{\mathrm{a}}$ Valores ajustados pelas outras variáveis; p: Nível descritivo do teste de associação do $\chi^{2}$. \# retirada da análise múltipla por perda da significância estatística; * não foi incluída na análise múltipla por apresentar $\mathrm{p}>0,20$ na análise univariada.

$O R^{b}$ : crude values; OR ${ }^{a}$ : Values adjusted by the other variables; $p$ : Descriptive level of the $\chi^{2}$ association test \# taken from multiple analysis due to the loss of statistical significance; * was not included in the multiple analysis because $p>0.20$ in the univariate analysis.

Dentre as variáveis relativas ao trabalho dos pais, apenas o trabalho da mãe durante a gravidez foi estatisticamente associado à ocorrência de cárie na análise univariada. ( $\mathrm{p}$ $=0,011)$ (Tabela 2 ).

Na Tabela 3 estão apresentadas as variáveis correspondentes à antropometria, às doenças e ao consumo de medicamentos ao longo da vida da criança, além de outros indicadores do crescimento e do desenvolvimento infantis. Nenhuma variável deste bloco mostrou-se associada à cárie dentária após o ajuste para as demais. Apesar de não se mostrarem associados à ocorrência de cárie, o sexo e o número de dentes presentes aos 12 meses foram mantidos na análise como variável de confusão.

No bloco de variáveis relativas aos hábitos e cuidados com a criança foram significativamente associados à ocorrência de cá- rie, após o ajuste: freqüentar pré-escola no sexto ano de vida $(\mathrm{p}<0,001)$, a época que a criança iniciou a escovação dentária e o consumo de doces (ambas com um $\mathrm{p}<0,025$ ) após o ajuste dentro do bloco. As crianças que não freqüentaram pré-escola no sexto ano de vida, que iniciaram tardiamente a escovação e que consumiam doces pelo menos uma vez ao dia, todos os dias, apresentaram OR $\left.\left[\mathrm{IC}_{95 \%} \mathrm{OR}\right)\right]$ elevados de $3,1(1,8$ $5,4), 1,7(1,1-2,8)$ e $1,9(1,2-3,0)$, respectivamente (Tabela 4$)$.

A análise de regressão logística múltipla hierarquizada mostrou os fatores de risco independentes para a ocorrência de carie (Tabela 5). No bloco mais distal de determinação foram fatores de risco para a ocorrência de cárie mães que estudaram 8 anos ou menos, $\left.\mathrm{OR}\left[\mathrm{IC}_{95 \%} \mathrm{OR}\right)\right]=2,0(1,2-3,3)$ e renda familiar entre 1,1 a 6 salários mínimos, 
Tabela 2 - Distribuição da proporção ponderada (\% p) da população segundo o ataque de cárie dentária (ceo-d $\geq 1$ ) e características do trabalho dos pais em crianças de 6 anos de idade. Pelotas, RS, Brasil, 1999.

Table 2 - Weighed proportion (\% $p$ ) of the population according to the dental caries index (ceo- $d \geq 1$ ) and characteristics of parental work in 6-year-old children. Pelotas, RS, Brasil, 1999.

\begin{tabular}{|c|c|c|c|c|c|}
\hline Variáveis/categorias & ceo-d $\geq 1 \% p$ & $\mathrm{OR}^{\mathrm{b}}\left[\mathrm{IC}_{95 \%}(\mathrm{OR})\right]$ & Nível P & $\mathrm{OR}^{\mathrm{a}}\left[\mathrm{I} \mathrm{C}_{95 \%}(\mathrm{OR})\right]$ & Nível P \\
\hline Trabalho mãe na gravidez & & & 0,011 & & 0,011 \\
\hline 8 a 9 meses & 48,1 & 1,0 & & 1,0 & \\
\hline 1 a 7 meses & 68,6 & $2,4[1,1-5,0]$ & & $2,4[1,1-5,0]$ & \\
\hline Não trabalhou & 65,9 & $2,1[1,2-3,4]$ & & $2,1[1,2-3,4]$ & \\
\hline Trabalho do pai ao nascimento da criança & & & 0,491 & & * \\
\hline Trabalhando & 61,0 & 1,0 & & * & \\
\hline Desempregado/afastado & 68,0 & $1,4[0,6-3,4]$ & & & \\
\hline Trabalho da mãe aos 12 meses da criança & & & 0,918 & & * \\
\hline Sim & 62,1 & 1,0 & & * & \\
\hline Não & 62,6 & $1,0[0,7-1,6]$ & & & \\
\hline Trabalho da mãe no $6^{\circ}$ ano de vida da crian & & & 0,227 & & * \\
\hline Todos os meses do último ano & 55,3 & 1,0 & & * & \\
\hline Alguns meses & 61,9 & $1,3[0,7-2,3]$ & & & \\
\hline Não trabalhou & 66,3 & $1,6[0,9-2,7]$ & & & \\
\hline
\end{tabular}

$\mathrm{OR}^{\mathrm{b}}$ : Valores brutos; $\mathrm{OR}$ : Valores ajustados pelas outras variáveis; p: Nível descritivo do teste de associação do $\chi^{2} ;{ }^{*}$ não foi incluída na análise múltipla por apresentar $\mathrm{p}>0,20$ na análise univariada.

$O R^{b}$ : crude values; ORa: Values adjusted by the other variables; $p$ : Descriptive level of the $\chi^{2}$ association test; ${ }^{*}$ was not included in the multiple analysis because $p$ > 0.20 in the univariate analysis.

$\left.\mathrm{OR}\left[\mathrm{IC}_{95 \%(} \mathrm{OR}\right)\right]=2,0(1,1-4,1)$ e igual ou menor que um salário mínimo, $\left.\mathrm{OR}\left[\mathrm{IC}_{95 \%} \mathrm{OR}\right)\right]=$ $3,0(1,3-7,0)$. Nenhuma variável relativa ao trabalho dos pais e ao período de crescimento e desenvolvimento infantil constituiu fator de risco para cárie. Dentre as variáveis relativas aos hábitos e cuidados verificou-se que não freqüentar a pré-escola no sexto ano de vida $\left.\mathrm{OR}\left[\mathrm{IC}_{95 \%} \mathrm{OR}\right)\right]=2,6(1,5-4,6)$ e consumir doce pelo menos uma vez por dia, todos os dias $\left.\mathrm{OR}\left[\mathrm{IC}_{95 \%} \mathrm{OR}\right)\right]=2,0(1,2-3,2)$ constituíram os principais fatores de risco, mesmo após o ajuste pelos blocos hierarquicamente superiores. O teste de Hosmer-Lemeshow ( $\mathrm{p}=0,948$ ) mostrou a adequação do modelo final, pois, conforme este teste, valores próximos a um indicam um bom ajuste do modelo.

\section{Discussão}

Existem poucos estudos epidemiológicos de coorte de nascidos vivos, de base populacional, que investigaram aspectos relativos à saúde bucal, sendo desconhecida a existência de algum deles no Brasil.
Houve a possibilidade de se ter uma clara definição cronológica dos eventos estudados, já que todos as informações sociais, antropométricas, médicas e relativas aos serviços de saúde foram coletadas ao nascimento, no primeiro, terceiro, sexto e décimo segundo meses e no sexto ano de vida, antes portanto da coleta de informações relativas à saúde bucal e ao exame odontológico realizados aos 6 anos de idade. $O$ desenho do estudo permitiu estabelecer claramente que as exposições estudadas ocorreram anteriormente ao efeito. Assim, foram evitados dois erros comuns em estudos epidemiológicos transversais ou caso-controle, que se referem à memória do entrevistado (recall bias) e à causalidade reversa. $\mathrm{O}$ sexo da criança $\mathrm{e}$ o número de dentes erupcionados aos 12 meses de idade foram controlados na análise estatística.

A população estudada foi obtida através de uma amostragem aleatória de todos os nascidos vivos em Pelotas no ano de 1993. A proporção de perdas, cerca de $10 \%$, pode ser considerada dentro dos parâmetros usuais para o tipo de estudo realizado. Mesmo 
Tabela 3 - Distribuição da proporção ponderada (\% p) da população segundo o ataque de cárie dentária (ceo-d $\geq 1$ ) e fatores de risco relacionados ao crescimento e desenvolvimento infantil. Pelotas, RS, 1999.

Table 3 - Weighed proportion (\% p) of the population according to the dental caries index (ceo- $d \geq 1$ ) and risk factors related to child growth and development. Pelotas, RS, Brasil, 1999.

\begin{tabular}{|c|c|c|c|c|c|}
\hline Variáveis/categorias & ceo-d $\geq 1 \% p$ & $\mathrm{OR}^{\mathrm{b}}\left[\mathrm{IC}_{95 \%}(\mathrm{OR})\right]$ & Nível P & $\mathrm{OR}^{\mathrm{a}}\left[\mathrm{IC}_{95 \%}(\mathrm{OR})\right]$ & Nível P \\
\hline Sexo & & & 0,624 & & 0,602 \\
\hline Masculino & 63,7 & 1,0 & & 1,0 & \\
\hline Feminino & 61,2 & $1,1[0,7-1,7]$ & & $0,9[0,6-1,4]$ & \\
\hline Peso ao nascer & & & 0,364 & & * \\
\hline Adequado ( $\geq 2500 \mathrm{~g})$ & 61,7 & 1,0 & & * & \\
\hline Baixo $(<2500 \mathrm{~g})$ & 69,4 & $1,4[0,7-3,1]$ & & & \\
\hline Altura/idade aos 12 meses & & & 0,126 & & $\#$ \\
\hline Adequada ( $\geq 2$ scores $Z$ ) & 61,8 & 1,0 & & $\#$ & \\
\hline Inadequada ( 2 scores Z) & 81,0 & $2,3[0,8-6,7]$ & & & \\
\hline Peso/Idade aos 12 meses & & & 0,901 & & * \\
\hline Adequada ( $\geq 2$ scores $Z$ ) & 62,5 & 1,0 & & * & \\
\hline Inadequada ( 2 scores Z) & 57,1 & $0,9[0,3-2,6]$ & & & \\
\hline Peso/altura aos 12 meses & & & 0,559 & & * \\
\hline Adequada ( $\geq 2$ scores $Z$ ) & 62,6 & 1,0 & & * & \\
\hline Inadequada ( 2 scores Z) & 33,3 & $0,4[0,0-4,6]$ & & & \\
\hline Dentes erupcionados aos 12 meses & & & 0,785 & & 0,686 \\
\hline$\leq 5$ & 63,8 & 1,0 & & 1,0 & \\
\hline$>5$ & 61,8 & $0,9[0,6-1,4]$ & & $0,9[0,6-1,4]$ & \\
\hline Hospitalização & & & 0,120 & & $\#$ \\
\hline Não & 65,8 & 1,0 & & $\#$ & \\
\hline $\operatorname{Sim}$ & 56,9 & $0,7[0,4-1,1]$ & & & \\
\hline Consumo de medicamentos do & & & 0,652 & & * \\
\hline \multicolumn{6}{|l|}{$1^{\circ}$ ao $6^{\circ}$ ano de vida } \\
\hline 0 a 2 & 63,2 & 1,0 & & * & \\
\hline 3 a 5 & 63,4 & $1,0[0,6-1,6]$ & & & \\
\hline 6 a 13 & 59,7 & $0,8[0,5-1,5]$ & & & \\
\hline Uso crônico de medicamentos do & & & 0,845 & & $*$ \\
\hline \multicolumn{6}{|l|}{$1^{\circ}$ a $6^{\circ}$ ano de vida } \\
\hline Não & 63,2 & 1,0 & & * & \\
\hline Sim & 58,1 & $0,8[0,4-1,5]$ & & & \\
\hline Amamentação no seio & & & 0,104 & & $\#$ \\
\hline$\geq 9$ meses & 65,9 & 1,0 & & $\#$ & \\
\hline 4 a 8,9 meses & 51,2 & $0,5[0,3-1,0]$ & & & \\
\hline 1 a 3,9 meses & 64,7 & $0,9[0,5-1,7]$ & & & \\
\hline$<1$ mês & 69,1 & $1,1[0,5-2,3]$ & & & \\
\hline Uso de mamadeira noturna & & & 0,747 & & * \\
\hline Não & 60,0 & 1,0 & & $*$ & \\
\hline Sim & 63,2 & $1,1[0,6-2,0]$ & & & \\
\hline
\end{tabular}

$\mathrm{OR}^{\text {b: }}$ Valores brutos; $\mathrm{OR}^{\mathrm{a}}$ : Valores ajustados pelas outras variáveis; $\mathrm{p}$ : Nível descritivo do teste de associação do $\chi^{2}$. \# retirada da análise múltipla por perda da significância estatística; * não foi incluída na análise múltipla por apresentar $p>0,20$ na análise univariada.

$O R^{b}$ : crude values; $O R^{a}$ : Values adjusted by the other variables; $p$ : Descriptive level of the $\chi^{2}$ association test \# taken from multiple analysis due to the loss of statistical significance; * was not included in the multiple analysis because $p>0.20$ in the univariate analysis. 
Tabela 4 - Distribuição da proporção ponderada (\% p) da população segundo o ataque de cárie dentária (ceo-d $\geq 1$ ) e fatores de risco relacionados aos cuidados com a criança, hábitos e consumo de doces, em crianças de 6 anos de idade. Pelotas, RS, 1999.

Table 4 - Weighed proportion (\% p) of the population according to the dental caries index (ceo- $d \geq 1$ ) and risk factors related to child care, habits and consumption of sweets in 6-year-old children. Pelotas, RS, Brasil, 1999.

\begin{tabular}{|c|c|c|c|c|c|}
\hline Variáveis/categorias & ceo-d $\geq 1 \% p$ & $\mathrm{OR}^{\mathrm{b}}\left[\mathrm{IC}_{95 \%}(\mathrm{OR})\right]$ & Nível P & $\mathrm{OR}^{\mathrm{a}}\left[\mathrm{IC} \mathrm{C}_{95 \%}(\mathrm{OR})\right]$ & Nível P \\
\hline Creche aos 6 meses & & & 0,109 & & \# \\
\hline Sim & 41,2 & 1,0 & & $\#$ & \\
\hline Não & 63,5 & $2,7[1,0-7,2]$ & & & \\
\hline Creche aos 12 meses & & & 0,099 & & $\#$ \\
\hline Sim & 42,1 & 1,0 & & $\#$ & \\
\hline Não & 63,7 & $2,3[0,9-5,9]$ & & & \\
\hline Pré-escola no $6^{\circ}$ ano de vida & & & $<0,001$ & & $<0,001$ \\
\hline Sim & 41,1 & 1,0 & & 1,0 & \\
\hline Não & 68,1 & $3,1[1,8-5,2]$ & & $3,1[1,8-5,4]$ & \\
\hline Consultou dentista no último ano (6 anos) & & & 0,399 & & * \\
\hline Sim & 61,2 & 1,0 & & * & \\
\hline Não & 66,7 & $1,3[0,8-2,1]$ & & & \\
\hline $\begin{array}{l}\text { Número de vezes que escova os } \\
\text { dentes ( } 6 \text { anos) }\end{array}$ & & & 0,019 & & $\#$ \\
\hline$\geq 3 \times$ ao dia & 56,4 & 1,0 & & $\#$ & \\
\hline 1 a $2 \mathrm{X}$ ao dia & 66,2 & $1,5[1,0-2,4]$ & & & \\
\hline$<1 \mathrm{X}$ ao dia & 81,5 & $3,2[1,2-8,8]$ & & & \\
\hline Criança recebe ajuda para escovar ( 6 anos) & & & 1,000 & & * \\
\hline Sim & 62,4 & 1,0 & & * & \\
\hline Não & 62,8 & $1,0[0,6-1,5]$ & & & \\
\hline Época que iniciou escovação & & & 0,010 & & 0,025 \\
\hline Qdo erupcionaram $1^{\text {os }}$ dentes & 52,9 & 1,0 & & 1,0 & \\
\hline Após 1 ano de idade & 67,7 & $1,8[1,2-2,9]$ & & $1,7[1,1-2,8]$ & \\
\hline Uso de fio dental aos 6 anos & & & 0,342 & & * \\
\hline Sim & 54,1 & 1,0 & & * & \\
\hline Não & 63,6 & $1,5[0,8-3,0]$ & & & \\
\hline Participou de ações educativas de & & 0,982 & & & * \\
\hline \multicolumn{6}{|l|}{ Saúde Bucal na escola } \\
\hline Sim & 62,0 & 1,0 & & * & \\
\hline Não & 62,7 & $1,0[0,7-1,6]$ & & & \\
\hline Consumo de doces aos 6 anos de idade & & & 0,011 & & 0,025 \\
\hline$<1 X$ ao dia & 53,2 & 1,0 & & 1,0 & \\
\hline$\geq 1 \mathrm{X}$ ao dia todo dia & 67,5 & $1,8[1,2-2,9]$ & & $1,9[1,2-3,0]$ & \\
\hline Costuma mascar chicletes aos 6 anos de id & & & 0,568 & & * \\
\hline Não & 61,7 & 1,0 & & * & \\
\hline Sim & 65,9 & $1,2[0,7-2,0]$ & & & \\
\hline Bebida + consumida aos 6 anos de idade & & & 0,565 & & * \\
\hline Água & 61,3 & 1,0 & & * & \\
\hline Outra & 65,3 & $1,2[0,7-1,9]$ & & & \\
\hline
\end{tabular}

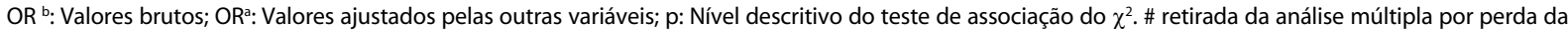
significância estatística; * não foi incluída na análise múltipla por apresentar $\mathrm{p}>0,20$ na análise univariada.

$O{ }^{b}$ : crude values; $O R^{a}$ : Values adjusted by the other variables; $p$ : Descriptive level of the $\chi^{2}$ association test \# taken from multiple analysis due to the loss of statistical significance; * was not included in the multiple analysis because $p>0.20$ in the univariate analysis. 
Tabela 5 - Resultados da análise de regressão múltipla hierarquizada para a ocorrência de cárie dentária em crianças de 6 anos de idade. Pelotas, RS, 1999.

Table 5 - Results of the hierarchy multiple regression analysis for dental caries in 6-year-old children.

Pelotas, RS, Brasil, 1999.

\begin{tabular}{|c|c|c|c|c|}
\hline Modelo & $\mathrm{OR}^{\text {bruta }}\left[\mathrm{IC}{ }_{95 \%}(\mathrm{OR})\right]$ & $\mathrm{p}^{\mathrm{b}}$ & 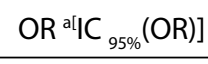 & $\mathrm{p}^{\mathrm{a}}$ \\
\hline \multicolumn{5}{|c|}{ Fatores socioeconômicos } \\
\hline Escolaridade da mãe & & $<0,001$ & & 0,012 \\
\hline$>8$ anos & 1,0 & & 1,0 & \\
\hline$\leq 8$ anos & $2,6[1,6-4,2]$ & & $2,0[1,2-3,3]$ & \\
\hline Renda familiar & & $<0,001$ & & 0,032 \\
\hline$>6 \mathrm{SM}$ & 1,0 & & 1,0 & \\
\hline 1,1 a 6 SM & $2,9[1,6-5,3]$ & & $2,1[1,1-4,1]$ & \\
\hline$\leq 1 \mathrm{SM}$ & $4,5[2,0-10,0]$ & & & \\
\hline
\end{tabular}

Fatores de crescimento e desenvolvimento

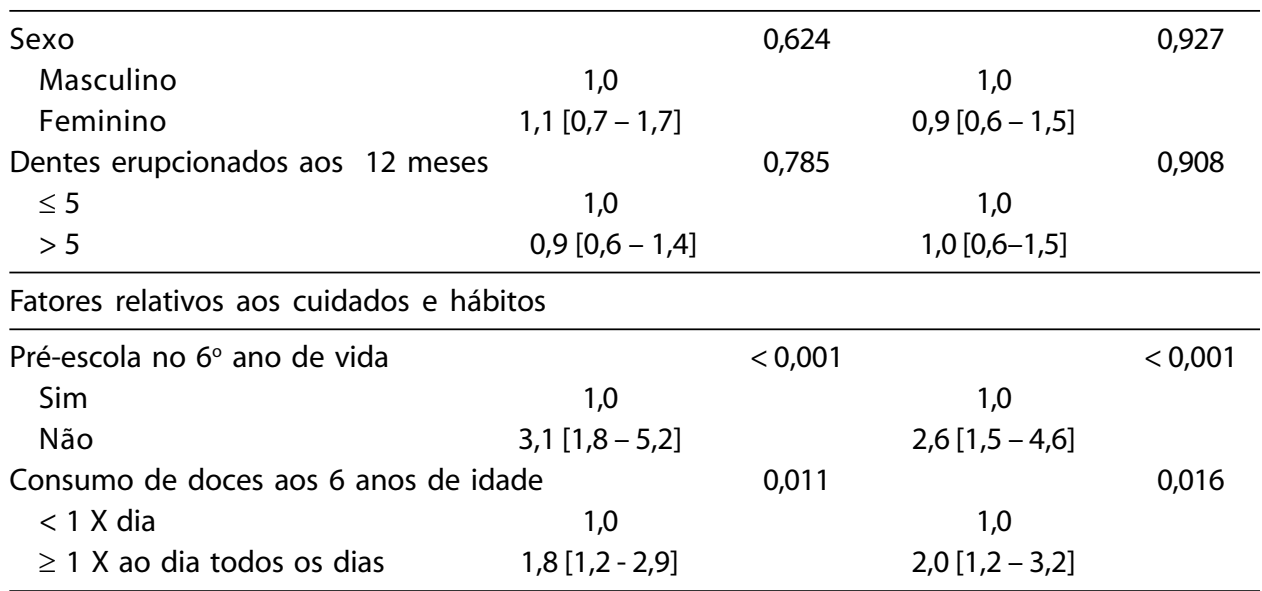

a: Ajustada internamente ao bloco e pelo(s) bloco(s) superior(es); b: análise univariada; Teste de Hosmer- Lemeshow $(p=0,948)$ a: Internally adjusted to block and by superior block(s); b: univariate analysis; Hosmer-Lemeshow Teste ( $p=0.948)$

acrescidas às perdas da fase anterior do estudo, de 6,6\%, o percentual de acompanhamento é elevado. Antes do estudo houve treinamento e calibração dos examinadores, registrando-se uma adequada concordância diagnóstica verificada no início e na fase intermediária do trabalho de campo. A forma aleatória da obtenção da amostra, o delineamento do estudo e a alta concordância diagnóstica entre os examinadores asseguraram a validade interna do estudo.

Por outro lado, deve-se tomar cuidado com as generalizações dos resultados. A população estudada é originária de uma cidade com indicadores sociais e de saúde acima dos padrões brasileiros. Estudos dirigidos às populações do Norte e Nordeste do Brasil são necessários para confirmar nossos achados.
As relações entre condições socioeconômicas e condições de saúde são bem estabelecidas e foram comprovadas neste estudo. Muitas medidas têm sido usadas para medir posição socioeconômica sendo que todas têm suas limitações e, portanto, tem sido recomendado utilizar pelo menos dois indicadores ${ }^{13}$.

Apesar desta recomendação, verifica-se que em muitos estudos acerca da relação saúde infantil e condições socioeconômicas das famílias não foram realizados ajustes estatísticos de um indicador pelo outro ${ }^{14}$. Outros pesquisadores, como Victora et al. ${ }^{15}$, analisando a influência do grau de educação da mãe em relação à mortalidade perinatal, infantil, hospitalizações, estado nutricional e doenças infantis observaram que a influ- 
ência da escolaridade materna sobre os desfechos mais imediatos, perinatais, é reduzida ou mesmo desaparece após ser ajustada por outras variáveis socioeconômicas, como renda familiar e escolaridade do marido. Entretanto, forte associação se mantém entre a escolaridade da mãe e desfechos mais tardios, mesmo depois de ajustada por outras variáveis socioeconômicas, o que confirma o importante papel da escolaridade da mãe na saúde de seus filhos.

Neste estudo foram consideradas as múltiplas e complexas dimensões socioeconômicas. Na análise univariada, todas as variáveis, com exceção daquelas relacionadas ao acesso à água, foram forte e significativamente associadas à ocorrência de cárie. Entretanto, após o ajuste pelas outras variáveis, dentro do mesmo bloco, apenas a educação das mães e a renda familiar permaneceram associadas significativamente.

O nível de educação é um importante marcador de condição socioeconômica e é útil, pois pode ser aplicado a ambos os sexos, ser usado para pessoas fora da força de trabalho, apresenta um comportamento estável ao longo da vida adulta e pode ser usado quando se compara diferentes regiões. Além do mais, altos níveis de escolaridade geralmente são preditores de melhores postos de trabalho, melhores condições de moradia e melhor posição socioeconômica ${ }^{13,16,17}$.

Possivelmente, um dos poucos campos onde é possível identificar estudos relativos à influência dos períodos perinatal e da primeira infância no desenvolvimento de cárie anos mais tarde são os estudos que abordaram o estado nutricional e cárie e erupção dentária, e aqueles que abordaram a relação entre hipoplasia de esmalte e sua associação com injúrias ocorridas muito precocemente como infecções e doenças. Alvarez et al. ${ }^{3}$ relataram retardo na erupção dentária e no pico de cárie em crianças desnutridas. Portanto, como existe um retardo no tempo de erupção nestas crianças, o número de dentes decíduos presentes deve ser controlado em estudos epidemiológicos a fim de evitar vieses. Assim, comparações entre países e regiões com diferentes prevalências de des- nutrição infantil devem ser realizadas com a devida cautela.

Os fenômenos que envolvem o processo desnutrição e cárie ocorrem em períodos em que vários dentes estão se desenvolvendo ao mesmo tempo, o que pode explicar a razão de estarem associados à alta severidade de cárie, mas não à ocorrência de alguma cárie $^{18}$.

O peso ao nascer, categoria chave nas teorias explicativas de todos os autores que trabalham com as teorias da programação biológica $^{5}$ e dos acúmulos de risco através $\mathrm{da} \mathrm{vida}^{6}$, não foi fator de risco para cárie, o que sugere fortemente que os fenômenos relacionados à cárie são fundamentalmente mais tardios, sociais e comportamentais, confirmando ser a cárie um fenômeno basicamente social, nutricional e dietético.

O papel desempenhado pela pré-escola foi importante para a ocorrência de cárie dentária. Este aspecto merece maiores investigações, pois não ficou claro se a influência da pré-escola como fator protetor de cárie foi devido às atividades relacionadas à educação em saúde bucal que lá ocorreram ou se o ambiente e a socialização da préescola levaram as crianças a terem comportamentos mais favoráveis à saúde bucal. Esta última hipótese parece ser mais realista, já que não houve associação entre cárie e a participação das crianças em atividades educativas e ou preventivas em saúde bucal na escola. Uma revisão sistemática da literatura mostrou a pouca eficácia de ações como palestras em escolas na melhoria da saúde bucal de crianças $^{19}$.

As variáveis relacionadas mais diretamente à odontologia, tais como acesso a consulta e aos métodos de higiene e prevenção bem conhecidos como os fluoretos, através da escovação, não foram associadas à carie de maneira independente. Estes achados não são surpreendentes, já que acesso ao dentista resulta em diminuição e alívio de dor e sofrimento, com conseqüente aumento na qualidade de vida, mas tem um papel pequeno na redução da cárie dentária. A pouca importância destas variáveis neste estudo pode se dever ao uso quase universal 
e regular de fluoreto via a água de abastecimento e dentifrícios nesta população. O uso quase universal destas medidas preventivas de eficácia comprovada pode explicar a ausência de associação, de maneira independente, com a ocorrência da cárie. Estas variáveis podem ser marcadores de condição socioeconômica. Com efeito, dados não apresentados revelaram que o uso de fio dental ( $\mathrm{p}<0,001)$, número de escovações dentárias $(\mathrm{p}<0,001)$ foram diretamente associados a rendas familiares mais elevadas e a escolaridade da mãe superior a oito anos ( $p<0,001$ e 0,022 respectivamente); o uso de fio dental foi associado a alta escolaridade do pai e a pertencer à classe situada no topo da hierarquia social $(\mathrm{p}<0,001)$. As variáveis "odontológicas" que se mostraram associadas na análise univariada mas perderam efeito após o ajuste revelam que as mesmas são confundidas pelas condições socioeconômicas, sendo estas "as causas das causas".

$\mathrm{O}$ último e mais proximal fator de risco investigado foi o consumo de doces. Pelotas é conhecida como a "capital brasileira do doce” e sua economia é dependente da manufatura e do comércio de doces, especialmente de tradição portuguesa, o que pode justificar o alto consumo da população. $\mathrm{O}$ padrão brasileiro de consumo de doces é um dos mais altos do mundo. Nas últimas déca- das houve um aumento para quase $15 \%$ da energia consumida ${ }^{20}$, quando o recomendável é no máximo $10 \%$ das calorias exigidas, 0 que equivale a cerca de 40 a 55 gramas ao dia ou 15 a $20 \mathrm{Kg}$ por ano ${ }^{21}$. O padrão de amamentação e o uso de mamadeira noturna não foram associados ao desfecho estudado.

Nosso estudo sugere que o enfoque na melhoria das condições de vida da população, com a conseqüente diminuição das desigualdades, aumento da renda familiar, escolaridade das mães, garantia do ingresso precoce da criança na escola e políticas de redução do consumo de açúcares seriam as principais estratégias para a prevenção da cárie dentária. $\mathrm{O}$ enfoque dirigido às causas comuns de doenças como a dieta, a higiene e a educação devem ser enfatizados.

Esta estratégia preventiva populacional deve ser complementada por serviços de saúde voltados para o alívio da dor e sofrimento e conseqüente melhoria da qualidade de vida.

\section{Agradecimentos}

Marco Aurélio Peres foi subvencionado com Bolsa de Estudos da CAPES e Karen Glazer com Bolsa de Estudos do CNPq. Dra. Latorre agradece ao CNPq a bolsa de pesquisadora.

\section{Referências}

1. Pan American Health Organization (PAHO). Oral Health. Directing Council. XL Meeting. Washington, DC; September 1997.

2. Horowitz HS. Research issues in early childhood caries. Community Dent Oral Epidemiol 1998; S 1: 67-81.

3. Alvarez JO, Caceda J, Woolley TW, Carley KW, Baiocchi N, Caravedo L, Navia JM. Longitudinal study of dental caries in the primary teeth of children who suffered from infant malnutrition. J Dent Res 1993; 72(12): 1573-6.

4. Seow WK. Biological mechanism of early childhood caries. Community Dent Oral Epidemiol 1998; 265 (S 1): 8-27.

5. Barker DJP. Mothers, Babies, and Disease in Later Life. London: BMJ Publishing Group; 1994.
6. Kuh D, Ben-Shlomo Y. A life course approach to chronic disease epidemiology. Oxford: Oxford University Press; 1997.

7. Sheiham A, Watt RG. The common risk approach: a rational approach for promoting oral health. Community Dent Oral Epidemiol 2000; 28: 399-406.

8. Victora CG, Barros FC, Tomasi E. et al. Tendências e diferenciais na saúde materno infantil: delineamento e metodologia das coortes de 1982 e 1993 de mães e crianças de Pelotas, Rio Grande do Sul. Cad Saúde Pública 1996; 12 (S 1): 7-14.

9. World Health Organization (WHO). Oral Health Survey. Basic Methods. Geneva, WHO; 1997. 
10. Lombardi C, Bronfman M, Facchini LA, Victora CG, Barros FC, Béria JU et al. Operacionalização do conceito de classe social em estudos epidemiológicos. Rev Saúde Pública 1988; 22(4): 253-65.

11. National Center for Health Statistcs, 1977. NCHS Growth Curves for Children. Birth-18 years. Rockville, MD: US Department of Health, Education and Welfare, publication no. (PHS) 79-1650.

12. Hosmer DW, Lemeshow S. Applied logistic regression. New York: Wiley; 1989.

13. Lynch J, Kaplan G. Socioeconomic position. In: Berkman L, Kawachi I. Social Epidemiology. New York: Oxford University Press; 2000. p. 13-35.

14. Brooks-Gunn J, Duncan GJ. The effects of poverty on children. The Future of Children. Children and Poverty 1997; 7(2): 55-71.

15. Victora CG, Huttly SRA, Barros FC, Lombardi C, Vaughan JP. Maternal education in relation to early and late child health outcomes: findings from a Brazilian cohort study. Soc Sci Med 1992; 34(8): 899905.
16. Kriger N, Williams DR, Moss NE. Measuring social class in US Public Health research: concepts, methodologies, and guidelines. Annu Rev Public Health 1997; 18: 341-78.

17. Manor O, Matthews S, Power C. Comparing measures of health inequality. Soc Sci Med 1997; 45(5): 761-71.

18. Peres MAA. Determinantes sociais e biológicos do período perinatal e da primiera infância na prevalência e severidade da cárie dentária em crianças de 6 anos de idade. \{Tese de Doutorado\}. São Paulo: Faculdade de Saúde Pública da USP; 2002.

19. Kay L, Locker D. Is dental health education effective? A systematic review of current evidence. Community Dent Oral Epidemiol 1996; 24: 231-5.

20. Monteiro CA, Modini L, Costa RBL. Mudanças na composição e adequação nutricional da dieta familiar nas áreas metropolitanas do Brasil (1988-1996). Rev Saúde Pública 2000a; 34(3): 251-8.

21. Pinto VG. Açúcares - suas relações epidemiológicas e econômicas com a cárie dental. In: Pinto VG. Saúde Bucal Coletiva. São Paulo: Santos; 2000b. p. 409.

Recebido em: 05/12/2002

Versão final reapresentada em: 23/07/2003 Aprovação em: 29/08/2003 